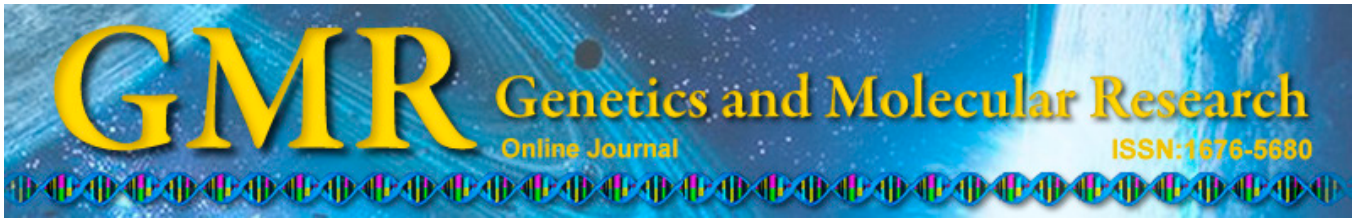

\title{
Hypoxia-inducible factor- $1 \alpha$ expression in the different stages of rat thromboangiitis obliterans
}

\author{
T. He*, B.H. Qu*, D.L. Wang and M. Hu \\ Department of Vascular Surgery, The Central Hospital of Wuhan, Wuhan, China \\ *These authors contributed equally to this study. \\ Corresponding author: T. He \\ E-mail: HeTao2013086@163.com
}

Genet. Mol. Res. 14 (2): 6715-6722 (2015)

Received August 14, 2014

Accepted January 26, 2015

Published June 18, 2015

DOI http://dx.doi.org/10.4238/2015.June.18.15

\begin{abstract}
We investigated the expression and effects of hypoxiainducible factor- $1 \alpha$ (HIF-1 $\alpha)$ in rat thromboangiitis obliterans (TO). Rats were divided into sham and model groups. The model group was further divided into groups based on observation duration. Lauric acid was injected below an artery clamp to simulate TO in the model group; saline was used in the sham group. Clamps were removed 15 min after injection in both groups, and physiological changes were observed at different times (gross observation and hematoxylin and eosin staining). The animals were killed at various times following the operation and serum and muscle tissues were sampled. For the sham group: the endometrium was relatively intact; medial membrane and epineurium lesions were absent; and blood vessels and surrounding tissues had no inflammatory cell infiltration. For the model group: all subgroups displayed inflammation; large numbers of inflammatory cells were gathered; muscle tissue lost its normal texture and structure; and the internal elastic membrane was integrated. Compared with the preoperative status, HIF-1 $\alpha$ expression increased significantly in all subgroups $(\mathrm{P}<0.05)$; there was no change in the sham group. HIF-1 $\alpha$ expression in each subgroup was different $(\mathrm{F}$ $=14.267, \mathrm{P}<0.05)$. Femoral artery injection of lauric acid can be used as
\end{abstract}


a rat TO model owing to its simple application and success rate. HIF-1 $\alpha$ expression increased in the early stage of $\mathrm{TO}$ and gradually decreased with the extension of ischemia time; it may play a leading role in TO development and can be used for diagnosis and cure evaluation.

Key words: HIF-1 $\alpha$; Thromboangiitis obliterans; Rats; Different stages

\section{INTRODUCTION}

Thromboangiitis obliterans (TO) is a common, chronic, recurrent segment inflammatory disease in small and medium-sized blood vessels. It usually appears in the limbs, small arteries and veins, and is most common in the lower limbs. The limb involved is often characterized by skin chill, reduced or undetectable pulse, numbness, and intermittent claudication accompanied by migratory thrombophlebitis or superficial phlebitis. Ulcers or necrosis appear in serious cases (Kothari et al., 2014; Liu et al., 2014a; Yuan et al., 2014). As a vascular occlusive disease, the main pathogenesis of TO is ischemia and hypoxia. Hypoxia-inducible factor- $1 \alpha(\mathrm{HIF}-1 \alpha)$ is an important transcriptional regulation factor that allows the body to adapt to low oxygen, and it participates widely in the adaptive response induced by hypoxia in mammalian cells (Yin et al., 2000; Wu et al., 2011; Tao et al., 2013). Some scholars have confirmed that HIF-1 $\alpha$ plays an important role in arteriosclerosis obliterans, but there is still no consensus on its role in the onset of TO (Tao et al., 2012). In the present study, HIF-1 $\alpha$ expression was investigated in TO rats at different times. We tried to provide theoretical data for evaluating the effect of HIF- $1 \alpha$ treatment on TO by regulating vascular endothelial growth factor (VEGF), and we explored the evaluation index for future early diagnosis and postoperative effect.

\section{MATERIAL AND METHODS}

\section{Animals}

We used 70 male Sprague Dawley rats weighing 280-320 g. All animals were raised and treated in strict accordance with the Animal Ethical Standard, and this study was approved by The Experimental Animal Center of Wuhan City, Hubei Province Ethics Committee.

\section{Reagents}

Lauric acid was bought from Daya Chemical (Tianjin, China); medical adhesive bandage was bought from Baiyun Medical Glue (Guangzhou, China); chloral hydrate was bought from Yulong Seaweed (Qingdao, China); penicillin (sodium salt) was bought from Youcare Pharmaceutical (Beijing, China); and enzyme-linked immunosorbent assay for HIF-1 $\alpha$ was obtained from Ann Inquiry (Shanghai, China).

\section{Instruments}

We used a paraffin slicing machine from the Nanbei Company (Zhengzhou, China). The ZS-2 enzyme-linked immunosorbent assay microplate reader was from Minxin Biotech- 
nology (Shanghai, China); the BX-51 LY-WN-HP-Super CCD was supplied by the Weizhun Company (Shanghai, China); the SH-5 heating magnetic stirrer was from the Ming Chi Company (Chengdu, China); the SC-3610 low-speed centrifuge was from the Zhongjia Company; the DH5000A incubator was from the Jinzhiye Company (Beijing, China); and the automatic washing machine was from Leidu Biotechnology (Shenzhen, China).

\section{Methods}

Seventy male Sprague Dawley rats were divided into sham operation (10 rats) and model (60 rats) groups. The model group was further divided into 1 day, 3 days, 1 week, 2 weeks, 3 weeks, and 4 weeks groups based on the duration of the observation period. The rats in the model group were anesthetized with $3 \mathrm{~mL} / \mathrm{kg} 10 \%$ chloral hydrate by intraperitoneal injection and fixed on the operating table. The hairs on the inner right thigh were removed and a 1.5-2.0 cm longitudinal incision was made. The femoral artery was freed and an artery clamp applied. Lauric acid $(0.2 \mathrm{~mL})$ was injected towards the heart from a place $1.0 \mathrm{~cm}$ below the artery clamp to simulate TO in the model rats, while $0.2 \mathrm{~mL}$ saline was used instead of lauric acid in the sham operation group. The artery clamp was removed 10 min after injection in both groups.

\section{Observational index}

The animals were killed at 1 day, 3 days, 1 week, 2 weeks, 3 weeks, and 4 weeks after the operation, and the serum and bilateral hind leg muscle tissues were sampled.

\section{Macroscopic observation}

Model success was determined by observing swelling, necrosis, gangrene, and muscle atrophy on the posterior limbs of the model animals.

\section{Hematoxylin and eosin $(\mathrm{H} \& \mathrm{E})$ staining}

For each group of rats, the posterior limbs were sampled, fixed with formaldehyde, embedded in paraffin, cut into slices, and stained with H\&E. The positive standard was red in the cytoplasm and violet in the nucleus.

\section{Serum enzyme-linked immunosorbent assay}

\section{Serum preparation}

Serum specimens were taken from the rats before and after modeling and preserved at $-20^{\circ} \mathrm{C}$. The specimens were diluted by sample diluent at $1: 2$ before testing.

\section{Standard solution preparation}

Distilled water $(2 \mathrm{~mL})$ was added to establish $80 \mathrm{ng} / \mathrm{mL}$ solutions. Eight standard tubes were set. The first tube specimen was diluted with $900 \mu \mathrm{L}$ diluent. The 2 nd- 8 th tube 
specimens were diluted with $500 \mu \mathrm{L}$ diluent. Standard solutions ( $100 \mu \mathrm{L}, 80 \mathrm{ng} / \mathrm{mL})$ were added to the first tube, and a $500 \mu \mathrm{L}$ mixed solution was transferred to the second tube. The dilution was repeated and the 8 th tube was set as a blank control.

\section{Testing}

The samples were tested on the microplate reader.

\section{Statistical analysis}

Measured data are reported as means \pm SD (standard deviation) and analyzed by ANOVA (analysis of variance) or LSD (least significant difference) t-test. All statistical analyses were performed using the SPSS 18.0 software (Chicago, IL, USA). P values of $<0.05$ were considered to be statistically significant.

\section{RESULTS}

\section{Macroscopic observation}

No obvious change appeared after the injection of saline solution to the femoral artery. The limbs affected appeared pale 3 min after injection of lauric acid, and limbs became bruised after about $40 \mathrm{~min}$. All the model rats showed claudication after $24 \mathrm{~h}$, accompanied by dragging, while the limb bruising became severe. Posterior limbs showed ischemia and swelling accompanied by black toes in the 1 day and 3 days groups. The ischemia was more serious and the ankles also displayed signs of necrosis in the 1 week group. Rats in the 2 weeks group had dry gangrene and the necrotic scope extended to the knees. Limb necrosis and the fall-off phenomenon were observed in the 3 and 4 weeks groups (Figure 1).
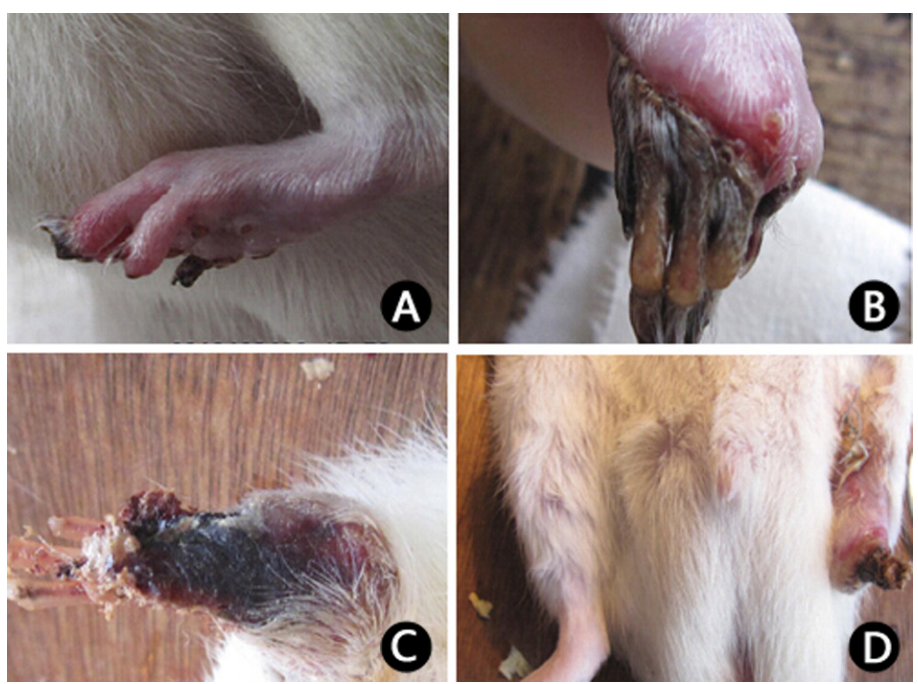

Figure 1. Different manifestations in the model rats. A. limb bruising and black toes. B. dry gangrene with swelling C. dry gangrene with bone exposed. D. limb necrosis and fall-off. 


\section{H\&E staining}

The sham operation group exhibited the following characteristics: the endometrium was relatively intact, without stripping or fall-off; there was an absence of lesions in the medial membrane and epineurium; and blood vessels and surrounding tissues had no inflammatory cell infiltration.

The model group exhibited the following characteristics: all subgroups displayed inflammation; large numbers of inflammatory cells were gathered; muscle tissue lost its normal texture and structure; and the pathological sections showed internal elastic membrane integration. There was segmental occlusion of the femoral artery in the 2, 3, and 4 weeks groups; thrombosis was obvious and a spate of new capillaries arose. Recanalization was visible in the vicinity of the thrombosis in the 3 and 4 weeks groups, while the pathological sections showed internal elastic membrane integration (Figure 2).

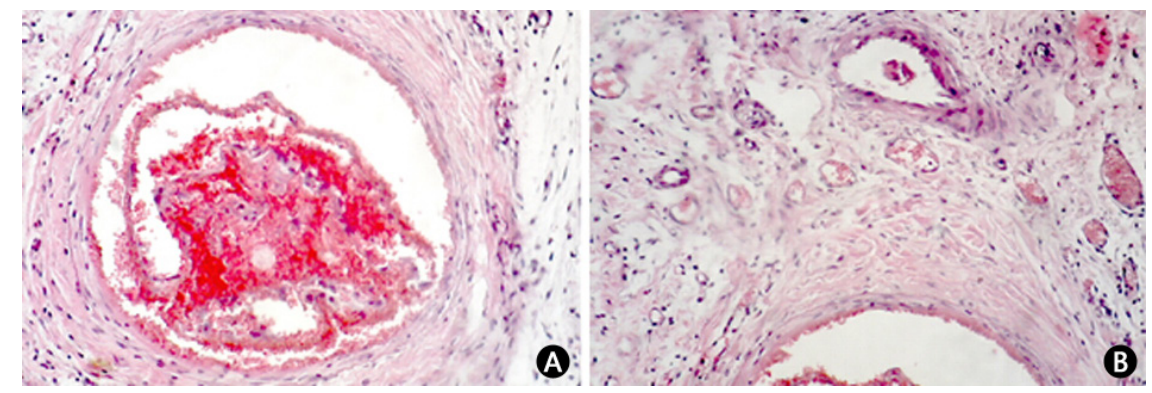

Figure 2. Thrombosis and inflammatory cell infiltration. (A) Arterial; (B) Venous [hematoxylin and eosin (H\&E), 400X]. A. Arterial thrombosis and inflammatory cell infiltration (H\&E, 400X). B. Venous thrombosis and inflammatory cell infiltration (H\&E, 400X).

\section{Serum HIF-1 $\alpha$ expression}

Compared with the preoperative status, HIF-1 $\alpha$ expression increased significantly in all the subgroups $(\mathrm{P}<0.05)$, but did not change in the sham operation group. HIF-1 $\alpha$ expression in each subgroup was different $(\mathrm{F}=14.267, \mathrm{P}<0.05)$, as shown in Figure 3 and Table 1.

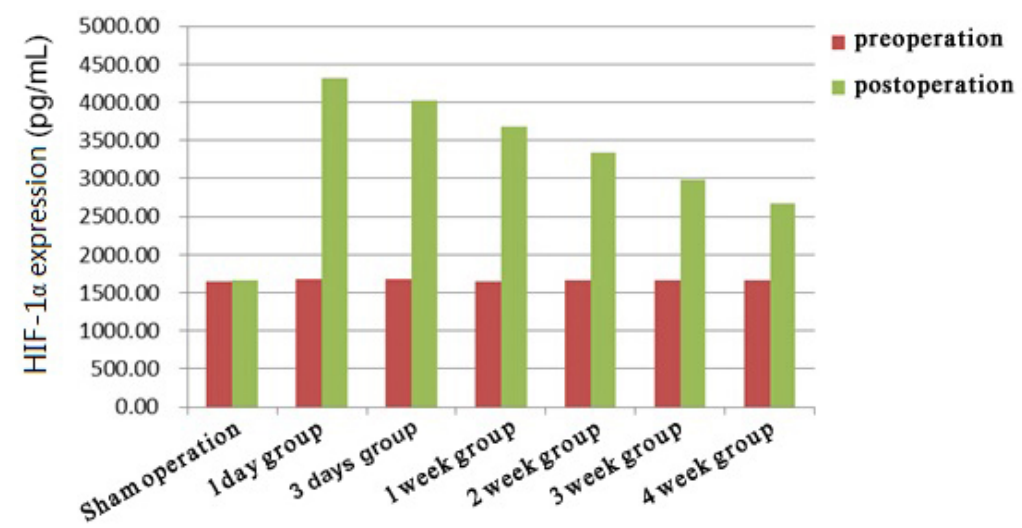

Figure 3. Comparison of serum HIF-1 $\alpha$ expression levels. 
Table 1. Serum HIF-1 $\alpha$ expression level (pg/mL).

\begin{tabular}{lcccrc}
\hline Group & No. of rats & Pre-operation & Post-operation & T value & P value \\
\hline Sham operation & 10 & $1645.6 \pm 23.4$ & $1660.0 \pm 27.5$ & 1.34 & $<0.05$ \\
1 day group & 10 & $1674.2 \pm 27.3$ & $4321.3 \pm 23.7$ & 30.03 & $<0.05$ \\
3 days group & 10 & $1677.0 \pm 33.4$ & $4024.5 \pm 24.9$ & 23.90 & $<0.05$ \\
1 week group & 10 & $1645.8 \pm 30.6$ & $3689.0 \pm 27.1$ & 15.09 & $<0.05$ \\
2 weeks group & 10 & $1665.3 \pm 29.8$ & $3345.2 \pm 23.0$ & 14.90 & $<0.05$ \\
3 weeks group & 10 & $1667.8 \pm 26.5$ & $2982.4 \pm 23.5$ & 13.83 & $<0.05$ \\
4 weeks group & 10 & $1670.01 \pm 24.4$ & $2673.7 \pm 27.4$ & 12.46 & $<0.05$ \\
\hline
\end{tabular}

\section{DISCUSSION}

Thromboangiitis obliterans is a type of chronic peripheral vascular occlusive disease. It commonly develops in young and middle-aged men, with characteristics of segmental, nonsuppurative inflammation in the small arteriovenous and arterial thromboses. Its nature is similar to ischemic atherosclerosis diseases of the limbs, but its pathogenesis and pathological behavior are different from those of other ischemic arterial diseases. There is lack of effective quantitative indicators for the treatment and diagnosis of TO (Chai et al., 2013; Pavlic et al., 2013; Yang et al., 2013; Fazeli et al., 2014; Ozbek et al., 2014; Ramin et al., 2014). HIF-1 $\alpha$ is the most important regulatory factor in hypoxic ischemia diseases (Tong et al., 2012), and it has wide potential in TO diagnosis, treatment, evaluation of operation effectiveness, and relapse monitoring.

HIF-1 protein was first extracted from hypoxia hepatocellular cancer cells Hep3b in 1992 (Kurata et al., 2000). HIF-1 comprises HIF- $1 \alpha$ and HIF-1 $\beta$, but the concentration of HIF1 is mainly decided by HIF-1 $\alpha$ (Liu et al., 2014b; Umar et al., 2014). As an important nuclear transcription factor, HIF-1 can activate the transcription of a variety of target genes under low oxygen conditions, and plays an important role in the processes of cell and tissue growth, and even physiological stress, in certain diseases (Feng et al., 2014). In the past 10 years, great progress has been made in understanding signal transduction pathways under hypoxia stress, especially in HIF-1 $\alpha$-mediated gene transcription regulation. In addition, HIF-1 can also regulate EPO and GLU T-1 genes to promote improvements in the conditions resulting from tissue hypoxia (An et al., 2014; Tao et al., 2014; Zhang et al., 2014). Some studies have suggested that HIF-1 activity can improve tissue recovery after ischemia (Yan et al., 2013; Zhang et al., 2013; Sun et al., 2014). The main mechanism that may explain this phenomenon is the participation of HIF-1 in gene overexpression of vascular system-related proteins, which increase levels of VEGF and its receptors in the vascular system and increase blood flow to relieve ischemia damage. Other studies (Baresić and Anić, 2014; Jun, 2014) have reported that collateral circulation reconstruction in arterial generation and endovascular blood flow increase were the most important and appropriate responses to the arterial occlusion. The present results also showed that HIF-1 $\alpha$ was upregulated in the early ischemic stage, while its level decreased gradually with the development of the disease. New capillaries and collateral circulation reconstruction could be found in the tissue 1 week after the operation, and the HIF$1 \alpha$ level was significantly higher than in the sham operation group. This indicates that low oxygen concentration can increase the expression of HIF- $1 \alpha$, and the expression of various angiogenesis factors increases more obviously, which strengthens the small blood vessels' ability to adapt to ischemia and hypoxia. Following disease progression, HIF-1 $\alpha$ levels decreased and made VEGF drop significantly, which failed to generate extensive effective collateral circula- 
tion, and led to a large degree of tissue necrosis, dry gangrene, and eventual limb loss.

In the present study, sodium laurate was used to prepare a TO animal model by inducing thrombosis through direct injury of the vascular endothelial cells. The thrombus formation within the blood vessels was rich in cellulose and platelets, owing to cellulose deposition, with occasional red blood cells. The effect was obvious and the method was successful. The pathologic analysis also shows that the elastic diaphragm was relatively integrated, a particularly clear indicator of atherosclerosis. Our study further confirmed that injection of sodium laurate to create a TO animal model can be used for TO pathogenesis research and exploration for new treatments.

HIF- $1 \alpha$ expression increased in the early stage of TO and gradually decreased with the extension of ischemia time. It may play a leading role in TO development and could be used as an indicator for stage diagnosis and curative effect evaluation. Femoral artery injection of lauric acid can be used to establish a rat TO model because it is simple and has a high success rate.

\section{REFERENCES}

An X, Xu G, Yang L, Wang Y, et al. (2014). Expression of hypoxia-inducible factor-1 $\alpha$, vascular endothelial growth factor and prolyl hydroxylase domain protein 2 in cutaneous squamous cell carcinoma and precursor lesions and their relationship with histological stages and clinical features. J. Dermatol. 41: 76-83.

Baresić M and Anić B (2014). Capillaroscopy in rheumatological practice - one center experience. Lijec Vjesn. 136: 28-32.

Chai DM, Bao ZQ, Hu JG, Ma L, et al. (2013). Vasculogenic mimicry and aberrant expression of HIF-1 $\alpha / E-c a d$ are associated with worse prognosis of esophageal squamous cell carcinoma. J. Huazhong Univ. Sci. Technol. Med. Sci. 33: 385-391

Fazeli B, Rafatpanah H, Ravari H, Farid Hosseini R, et al. (2014). Sera of patients with thromboangiitis obliterans activated cultured human umbilical vein endothelial cells (HUVECs) and changed their adhesive properties. Int. J. Rheum. Dis. 17: 106-112.

Feng L, Tao L, Dawei H, Xuliang L, et al. (2014). HIF-1 $\alpha$ expression correlates with cellular apoptosis, angiogenesis and clinical prognosis in rectal carcinoma. Pathol. Oncol. Res. 20: 603-610.

Jun HJ (2014). Endovascular revascularization for the obstruction after patch angioplasty in Buerger's disease. Korean J. Thorac. Cardiovasc. Surg. 47: 174-177.

Kothari R, Sharma D, Thakur DS, Kumar V, et al. (2014). Thoracoscopic dorsal sympathectomy for upper limb Buerger's disease. JSLS. 18: 273-276.

Kurata A, Franke FE, Machinami R and Schulz A (2000). Thromboangiitis obliterans: classic and new morphological features. Virchows. Arch. 436: 59-67.

Liu Q, Zhou HC, Yang XL, Zhao YP, et al. (2014a). Embolus-carried vascular endothelial cell growth factor 165 improves angiogenesis in thromboangiitis obliterans. Genet. Mol. Res. 13: 1744-1752.

Liu J, Chen JD, Nie P, Sheng X, et al. (2014b). Expression of HIF-1 $\alpha$ in the genioglossus associated with induced bilateral intermittent nasal obstruction in young rats. Shanghai Kou Qiang Yi Xue. 23: 138-142.

Ozbek C, Kestelli M, Bozok S, Ilhan G, et al. (2014). Surgical stimulation of angiogenesis. Asian Cardiovasc. Thorac. Ann. 22: 36-39.

Pavlic V, Vujic-Aleksic V, Zubovic N and Gojkov-Vukelic M (2013). Periodontitis and Buerger's disease: recent advances. Acta. Inform. Med. 21: 250-252.

Ramin M, Salimi J and Meysamie A (2014). An Iranian scoring system for diagnosing Buerger's disease. Acta. Med. Iran. 52: 60-65.

Sun L, Wu L, Qiao Z, Yu J, et al. (2014). Analysis of possible factors relating to prognosis in autologous peripheral blood mononuclear cell transplantation for critical limb ischemia. Cytotherapy 16: 1110-1116.

Tao BB, Zhang CC, Liu SY and Zhu YC (2012). Involvement of HIF-1 in the migration-promoting effects of hydrogen sulfide in vascular endothelial cells under normoxic conditions. Sheng Li Xue Bao. 64: 129-134.

Tao J, Doughman Y, Yang K, Ramirez-Bergeron D, et al. (2013). Epicardial HIF signaling regulates vascular precursor cell invasion into the myocardium. Dev. Biol. 376: 136-149.

Tao LL, Shi SJ, Chen LB and Huang GC (2014). Expression of monocyte chemotactic protein-1/CCL2 in gastric cancer and its relationship with tumor hypoxia. World J. Gastroenterol. 20: 4421-4427.

Tong JJ, Yan Z, Jian R, Tao H, et al. (2012). RhoA regulates invasion of glioma cells via the c-Jun NH2-terminal kinase

Genetics and Molecular Research 14 (2): 6715-6722 (2015)

CFUNPEC-RP www.funpecrp.com.br 
pathway under hypoxia. Oncol. Lett. 4: 495-500.

Umar A, Zhou W, Abdusalam E, Tursun A, et al. (2014). Effect of Ocimum basilicum L. on cyclo-oxygenase isoforms and prostaglandins involved in thrombosis. J. Ethnopharmacol. 152: 151-155.

Wu S, Cheng Z, Yu L, Song W, et al. (2011). Expression of CD82/KAI1 and HIF-1alpha in non-small cell lung cancer and their relationship to vasculogenic mimicry. Zhongguo Fei Ai Za Zhi. 14: 918-925.

Yan J, Liu Q, Dou Y, Hsieh Y, et al. (2013). Activating glucocorticoid receptor-ERK signaling pathway contributes to ginsenoside Rg1 protection against $\beta$-amyloid peptide-induced human endothelial cells apoptosis. J. Ethnopharmacol. 147: 456-466.

Yang X, Yang B, Cai J, Zhang C, et al. (2013). Berberine enhances radiosensitivity of esophageal squamous cancer by targeting HIF-1 $\alpha$ in vitro and in vivo. Cancer Biol. Ther. 14: 1068-1073.

Yin JH, Yang DI, Ku G and Hsu CY (2000). iNOS expression inhibits hypoxia-inducible factor-1 activity. Biochem. Biophys. Res. Commun. 279: 30-34.

Yuan L, Bao J, Zhao Z, Lu Q, et al. (2014). Clinical results of percutaneous transluminal angioplasty for thromboangiitis obliterans in arteries above the knee. Atherosclerosis 235: 110-115.

Zhang C, Yang X, Zhang Q, Yang B, et al. (2014). Berberine radiosensitizes human nasopharyngeal carcinoma by suppressing hypoxia-inducible factor-1 $\alpha$ expression. Acta. Otolaryngol. 134: 185-192.

Zhang Y, Jiang YR, Lu Q, Yin H, et al. (2013). Apelin in epiretinal fibrovascular membranes of patients with retinopathy of prematurity and the changes after intravitreal bevacizumab. Retina. 33: 613-620. 